\title{
Addressing Mental Health Stigmas among Refugees: A Narrative Review from a Socio-Ecological Perspective
}

\author{
Huaibo Xin \\ Department of Applied Health, Southern Illinois University Edwardsville, USA \\ Received November 20, 2019; Revised January 7, 2020; Accepted February 3, 2020
}

Copyright $\bigcirc 2020$ by authors, all rights reserved. Authors agree that this article remains permanently open access under the terms of the Creative Commons Attribution License 4.0 International License

\begin{abstract} predominantly prevalent among different refugee populations resettled in both western and non-western countries. It significantly affects refugees' mental healthcare seeking behaviors. The current narrative review is to examine the anti-stigma strategies/ interventions/ programs that specifically address refugees' mental health stigma. Twenty-six out of 711 peer-reviewed journal articles, book chapters, commentaries, reports, dissertations, and theses published in 2019 or prior in English were included in the final themes analysis. Results were organized using a socio-ecological multilevel framework (intrapersonal, interpersonal, institutional, community, and policy levels), which include increasing refugee individuals' awareness of mental health and improving their attitudes and perceptions towards mental disorders; enhancing interpersonal interactions between refugee patients and people who can encourage them to seek mental healthcare and can provide them with culturally competent mental health services; delivering mental healthcare through schools and ethnic organizations; developing community-based bottom-up interventions and mobilizing community assets to destigmatize and normalize mental illnesses within refugee communities; and advocating for a change of social norm towards mental illnesses and mental health treatment. There is a significant lack of studies designed to explore the effectiveness of anti-stigma approaches among refugees, which leads to an urgency of developing these studies and supporting evidence-based practice.
\end{abstract}

Keywords Stigma, Refugees, Mental Health, Socio-Ecological Model

\section{Introduction}

There was a rich body of literatures discussing mental health stigma among refugee populations over the past at least 40 decades [1-7]. The conversation has continued in the most recent 10 years [8-10]. Mental health related stigma consistently presents itself as one of the major barriers to the utilization of mental health services among a variety of western or non-western refugees [8-10]. In 2010, a national survey was sent out to 49 state refugee health coordinators in the U.S. to examine the practice of screening for war trauma, torture, and mental health symptoms among refugees, as well as to identify the gaps for refugees to access mental health screening and treatment services. Results showed that approximately, $76 \%$ of 41 state health coordinators reported that mental health stigma was one of the major barriers to accessing mental health services. Compared to the states who did not provide refugees with mental health screening, states who provided refugees with screening services were more likely to report stigma as a barrier [8]. Two systematic reviews published in 2014 and 2015 suggested that because of social stigma attached to mental illnesses, refugees chose to express their mental health symptoms as somatic symptoms [9, 10]. In Rohlof et al. review, they explicitly pointed out that refugee patients "consciously or unconsciously preferred to present somatic problems rather than psychological problems because of fear of being called insane by their doctors, their family, or others" [10]. Patients showed greater fear of being stigmatized and more likely hid their psychiatric symptoms if he/she came from a country where people understood psychiatry as a care system only for schizophrenics [10].

Mental health stigma widely exists among different refugee populations [11-17]. A study was conducted to compare the self-reported mental health status between US-born and Somalia-born Black Americans and White Americans [11]. Somalis were more likely to report embarrassment about seeing a mental health professional than the rest of the respondents in the study. About 24\% of Somali respondents felt that it would be very embarrassing to tell their friends that they were seeking 
mental healthcare while there were fewer than $5 \%$ of US-born Black respondents expressed the same concern in the study. Overall, Somalis reported the highest levels of mental health stigma [11]. A similar theme emerged among 20 Somali adults in Sweden. Participants believed that mental illnesses led to stigma and risk of social isolation [12]. Somalis with mental illnesses were treated badly by the public, and were taunted by children on the streets [13]. A total of 97 Australians, Iraqis, and Sudanese living in Australia participated in a study exploring the differences in mental health knowledge and beliefs among these three populations. Compared to the Australian participants, the Sudanese indicated significantly more stigmatizing attitudes towards patients with post-traumatic stress disorder while the Iraqis expressed significantly more stigmatizing attitudes towards patients with depression [14]. Likewise, mental health related stigma in Syrian society remained very high, which resulted in Syrians hesitating to receive mental health screening and treatment and suffering in silence [15]. Southeast Asian refugee elders clearly stated that they would not share their mental health issues with anyone outside of their family due to the fear of stigma [16]. Unsurprisingly, peer disapproval of seeking professional mental health services, such as seeing a school counsellor, was also prevalent among refugee adolescents [17, 18]. Refugee adolescents tended to equalize mental illnesses to craziness, retarded, weird, and abnormal, and perceived mental illnesses as a source of shame, which should not be disclosed for help [17]. Mental healthcare professionals further emphasized that mental illness was deeply stigmatized in refugee communities, and people from countries outside of U.S. often held a very different view on mental health. Mental healthcare professional could easily be depreciated and mistrusted $[19,20]$. The purpose of this paper is to examine the strategies/programs/interventions that have been adopted to address mental health related stigmas among refugee populations through a narrative systematic review, and identify the directions for future endeavor.

\section{Materials and Methods}

\subsection{Inclusion and Exclusion Criteria}

Publications in English (e.g., peer-reviewed journal articles, commentaries, books, book chapters, reports, theses, and dissertations) related to reduction of mental health stigma among any refugee populations in the U.S. and any other countries were included. Anti-stigma approaches could be anything from a simple strategy to a well-designed intervention/prevention study. All included publications are published in 2019 or prior either quantitatively or qualitatively. Information related to immigrants and asylum were excluded from the current review.

\subsection{Search Strategy}

A total of 711 peer-reviewed journal articles, books, book chapters, reports, commentaries, thesis, and dissertation in English were included in the initial search. Several major data databases (e.g., PubMed, MEDLINE, EBSCOhost, PsycINFO, Google Scholar) and three key phrases "stigma + mental health + refugees" were used for this search. Among these 711 publications, 26 peer-reviewed journal articles and one report were chosen for the final data analysis. All of them described or discussed at least either an approach or an intervention/prevention study to preventing mental health stigma among refugees.

\subsection{Data Extraction and Synthesis}

Theme analysis was adopted to analyze the information extracted from the 26 peer-reviewed journal articles and report. Codes and subcategories were generated to capture and categorize the information from these 26 publications related to the ways of minimizing mental health stigma among refugee populations. In the end, subcategories emerged during the analysis were further categorized into five levels of intervention/prevention strategies to addressing refugees' mental health stigma, including intrapersonal, interpersonal, institutional, community, and policy levels within the socio-ecological model.

\section{Results}

All 26 publications were published between 1991 and 2018. Qualitative data extracted from these publications were categorized into five levels using the socio-ecological model.

\subsection{Intrapersonal Level}

Both health providers and refugees recognized the needs of increasing refugee individuals' knowledge of mental health and changing their attitudes towards mental disorders in order to fight with mental health stigma [21-24]. The Mental Health First Aid program was particularly designed to "increase mental health literacy, decrease stigmatizing attitudes, and prepare community members to recognize and assist individuals who are in crisis" [22]. Refugee participants in Donnelly et al. study indicated that there was a need of creating and disseminating culturally competent educational materials for mental health through culturally appropriate channels among refugee populations. Participants believed that increasing refugee communities' awareness of mental disorders would help "reduce social stigma and discrimination towards mental illnesses" [24].

\subsection{Interpersonal Level}

The current literatures suggested that it could 
potentially decrease the stigmatization among refugee populations and increase their acceptance of and access to mental healthcare through a variety of human interactions between the patients and their family members, partners, colleagues, friends, service providers, as well as their community members [25-36]. Interventions can be adopted to deliver tailored educational programs and anti-stigma information to people who are close and relevant to refugee patients and can eventually help them overcome the barriers and receive the mental healthcare [25-26]. Physicians can also educate their refugee patients about mental well-being during their primary care visits so that their patients "may be relieved to know that symptoms of post-traumatic stress disorder and major depression are common and treatable responses to trauma" [27], and realize that "mental health services are 'professional' like physical doctors and will be likely to supply relief rather than bring shame and stigma" [28]. Health providers could focus more on the resilient aspect of mental health and emphasize individuals' resilient properties, such as "strength, knowledge, and skills" [29], which can be used to overcome mental illnesses. It has proven to be effective that mental healthcare providers avoid using terms (e.g., mental illness, psychiatric) to describe mental disorders in order to "prevent unnecessary stigmatization [30, 31]. It is critical to build trusting relationships between mental health service providers and refugee patients. It creates a safe environment for refugees to open themselves up in addressing psychological issues during their medical visits [32]. The current literatures also recommend that a mental health service provider do an initial assessment in the home of a client who was not willing to go to a mental health clinic because of the fear of being stigmatized. [33]. As alternative therapies, interventions to develop targeted social support groups, including "coffee mornings, sewing groups, art groups" [35], and women groups were supported by refugees to reduce their fear of mental stigma [36].

\subsection{Organizational/Institutional Level}

Themes emerged from the current literatures demonstrated the effectiveness of reducing the levels of mental health stigmatization among refugees through institutional preparation of a culturally competent mental health workforce and delivery of tailored mental health services $[23,25,26,35,37-43]$. It is crucial for agencies to train their mental healthcare providers to recognize and understand the fear and stigma associated with mental disorders and attached to refugee individuals and their communities $[23,25]$. Given the prevalence of mental health issues among refugee adolescents, it would be very helpful for mental healthcare services being integrated into a school system to enhance the confidentiality and normalization of the disease and lower the possibility of patients' being stigmatized [26, 37-39]. Ellis et al. indicated in their review that "locating services within service systems that are trusted and highly accessed by refugee families and youth, such as schools, is a powerful approach to diminishing the stigma associated with mental health services" [37]. An additional approach is to integrate culturally competent mental health services into local community organizations or community clinics that work closely with refugees [35, 40-43]. A Kurdish organization in London hired bilingual and bicultural health workers who were trained in transcultural mental health and were able to "build up a trusting relationship with service users and alleviates much of the stress, stigma and alienation associated with accessing the current mainstream health and social care services" [35]. Miller found out that by training English as a Second Language (ESL) teachers about refugees' experience in distress, it provided refugees with a safe platform to share and address their psychological needs in their ESL classroom [40]. Several community mental health clinics in California and Minnesota incorporated culturally appropriate services. They included acupuncture, and hired a Buddhist monk who had formal social work training, in order to reduce psychiatric stigma and enable the mental healthcare access for their refugee clients [41].

\subsection{Community Level}

It has been repeatedly emphasized that community engagement and mobilization is central to the destigmatization of mental disorders among refugee populations $[25,26,29,35,36,44-46]$. As more community members learn about mental illnesses and their treatment, it increases the chances of normalizing the disease within the community and providing the neighbors, friends, peers, family members, etc. who are suffering from the disease with more social support. For example, Schuchman and McDonald indicated that "the many community presentations made in Minnesota have had a noticeable impact on the destigmatization of mental illness and the acceptance of Western mental health treatment" [45]. Ao et al. stated that engaging refugee communities could bring "considerable strengths and cultural knowledge of refugee community members to bear on the issues", such as mental health stigma. They believed that refugees themselves were active change agents in a process like removing mental health stigmas [36]. Johnson-Agbakwu et al. demonstrated that "there is a need for community-partnered, culturally tailored interventions to provide health promotion education, dispel myths, and reduce the stigma of mental health, while accentuating (community) asset-based, strength models of resiliency and community social support" [44]. Collaborating with refugee communities and contracting community partners was "to get cultural expertise and buy-in from the community they represent, to help mitigate the shame and stigma about mental health prominent among new arriving populations" [46]. Community partners could help developing 
psycho-educational events, including cultural shows incorporating cultural music and dance as a means to educate community members, increase community awareness, and destigmatize mental illness and mental health treatment [46].

\subsection{Policy Level}

Advocating for a change of social norm towards mental illnesses could also help with the destigmatization [45]. For instance, Schuchman and McDonald indicated in their study that "Somalis often needed advocacy within their family and community in order to reduce stigma and prevent social isolation" [ 45].

\section{Discussion}

In the current review, five level strategies were identified to address mental health stigmas among refugees resettled in different countries - (i) delivering culturally appropriate educational interventions to refugee individuals and improving their mental health literacy and changing their attitudes and perceptions towards mental disorders; (ii) relying on human interactions between refugee patients and their families, friends, co-workers, primary healthcare providers, and neighbors who know about mental disorders and hold a positive view on them, to support and encourage refugee patients to seek mental healthcare; (iii) building a culturally competent mental healthcare workforce who understands refugees' fear and stigma of mental illnesses as well as their cultural interpretation of psychological/psychiatric concerns, and can provide refugees with culturally competent services; (iv) utilizing schools and community/ethnic organizations to assure refugee patients an easy and safe access to mental healthcare; (v) mobilizing community assets and collaborating with community partners to increase community's awareness of mental illnesses and psychological/psychiatric treatment and to destigmatize and normalize mental disorders within refugee communities; (vi) advocating for a change of social norm towards mental illnesses [21-45]. Although there is a significant lack of studies examining the effectiveness of these strategies among refugee populations as indicated by a recent review of anti-stigma interventions in Canada, that most of the participants in these interventions were White, Canadian-born, youth and/or middle-aged women instead of immigrants, people of diverse ethnic backgrounds, and older adults [47]. These strategies have been consistently utilized among general populations in the mainstream of the society [47-74].

The effectiveness of anti-stigma interventions among general populations were very well researched employing study designs like randomized controlled trials, quasi-experimental studies, and cohort studies, as well as a variety of psychosocial interventions like Cognitive Behavior Therapy, psychoeducation, cognitive restructuring, narrative enhancement, social skills training [47-74]. A recent narrative review of systematic reviews and primary research studies summarized the impact of anti-stigma interventions and indicated that population-based anti-stigma interventions led to some consistent short-term positive changes in individuals' attitudes and knowledge [49]. A meta-analysis of 19 educational randomized controlled trials concluded that educational interventions through either online or in-person delivery were effective in reducing personal stigma, i.e. an individual's attitude towards people with a mental disorder [71]. A systematic review and meta-analysis of 20 educational randomized controlled trials concluded that overall, these interventions led to small-to-medium reductions in stigmatizing attitudes [69]. Anti-stigma interventions were also proven to be effective among target population groups, such as employees and college students, in improving their knowledge and attitudes towards mental health patients, and the use of mental healthcare services [61, 67]. Medium and long-term effect (i.e. 4 weeks or more after an intervention was completed) in increasing mental health knowledge and decreasing discriminatory attitudes towards mental illnesses was found among 80 quantitative studies conducted globally [68].

Contact-based interpersonal anti-stigma education programs have also been heavily discussed especially among the general youth population $[55,57,60]$. As defined by Chen et al., contact-based education involved people with lived experience of mental illnesses in sharing their stories with other individuals and/or their families and delivering positive messages about recovery [55]. Chen et al., developed a school-based and contact-based program model for high-school students with an overarching theme of "engaging contact reduces stigma" and three focus areas of "connection, engagement, and empowerment" [55]. As suggested by an evaluation of 21 contact-based education programs in 5,047 Canadian high school students, these programs were promising for reducing stigma among high-school students [60]. In the meantime, a study was conducted to assess the effectiveness of population-based mass social contact interventions among people with or without mental health concerns in "improving intended stigmatizing behavior, increasing future willingness to disclose a mental health problem, and promoting behaviors associated with anti-stigma campaign engagement" [58]. The study demonstrated the effectiveness of these types of interpersonal approaches as well as suggested the likelihood of constructively facilitating inter-group contact and understanding between people with and without mental health concerns [58].

Amongst the refugee studies, studies done with the general population also supported the anti-stigma 
approaches that are beyond individual and group levels and at the institutional/organizational and community levels [50, 52-54]. For example, an anti-stigma educational intervention was built into the first-year nursing curriculum to decrease first-year nursing undergraduate students' stigmatizing beliefs and attitudes. Through guided clinical practice, discussion focusing on attitudes and beliefs towards mental health patients was carried out with first-year nursing students. The findings from this study suggested a positive implication for institutional curriculum change in mental health content for undergraduate nursing students [53]. At the community level, as part of the 2009-2014 "Time to Change" anti-stigma program, utilizing mass media, social media, and social contact events, a social media campaign was delivered to middle income people aged between mid-twenties and mid-forties. The campaign addressed the target population's mental health knowledge, and their attitudes and behaviors towards people with mental illnesses, and was proven to have a wide-ranging effect on challenging stigma [52]. Moreover, like Knifton et al. study, they also used community-based approaches to address mental health stigma. Community organizations designed and delivered 26 mental health awareness workshops. These workshops acknowledged cultural constructs of mental health, and were very well received by community groups. The study argued that community-based bottom-up anti-stigma interventions were more effective than top-down programs [50]. Best practice or evidence-based practice among general populations may be adaptable and replicable among refugees resettled in their host countries. However, there are cross-cultural differences in stigma [48]. Public stigmatizing attitudes could vary across different categories of mental disorders; could vary across populations/countries with different socio-economic status; and could vary across people with different religions [48]. This further demonstrates the needs of developing interventions and assessment plans to examine the effectiveness of anti-stigma strategies particularly among refugee populations. In order to meet the needs, it is essential to establish partnerships between researchers and refugee communities to identify the best practice in refugees' anti-stigma programming, replicate it, and expand it. The partnerships can also serve as bridges among researchers, policymakers, healthcare providers, community practitioners, and refugees to further enhance the likelihood of evidence-based practice and advocate for anti-stigma mental health policies [48].

\section{Conclusions}

There have been multilevel and multifaceted approaches to address mental health related stigmas among both refugee populations and general populations
[21-74]. Although many of these approaches have been proven effective and feasible among general populations, they could not be much effective among refugees [47]. The efficacy, feasibility, effectiveness, and replicability of anti-stigma programs often vary by populations as well as categories of mental illnesses [48]. Given the high prevalence and incidence of mental disorders and the significance of mental health stigmas among refugees [1-17], there is an urgency to design and deliver more culturally competent refugee-specific anti-stigma intervention studies, examine their effectiveness, and facilitate more evidence-based practice.

\section{REFERENCES}

[1] K. Harris, C. Maxwell. A needs assessment in a refugee mental health project in North-East London: Extending the counselling model to community support. Medicine, Conflict and Survival, Vol. 16, No. 2, 201-215. https://www.tandfonline.com/doi/abs/10.1080/1362369000 8409514

[2] W. Rutz. Mental health in Europe: Problems, advances and challenges. Acta Psychiatrica Scandinavica, Vol. 104, 15-20.

https://onlinelibrary.wiley.com/doi/full/10.1034/j.1600-04 47.2001.1040s2015.x

[3] E. Hsu, C. A. Davies, D. J. Hansen. Understanding mental health needs of Southeast Asian refugees: Historical, cultural, and contextual challenges. Clinical Psychology Review, Vol.24, No. 2, 193-213.https://www.sciencedirect.com/science/article/pii/ S0272735803001491

[4] H. Fenta, I. Hyman, S. Noh. Mental health service utilization by Ethiopian immigrants and refugees in Toronto. The Journal of Nervous and Mental Disease, Vol. 194, No. 12, 925-934.https://journals.lww.com/jonmd/Fulltext/2006/12 000/Determinants_of_Depression_Among_Ethiopian.7.asp $\mathrm{X}$

[5] M. Shoeb, H. Weinstein, R. Mollica. The Harvard trauma questionnaire: Adapting a cross-cultural instrument for measuring torture, trauma and posttraumatic stress disorder in Iraqi refugees. International Journal of Social Psychiatry, Vol.53, No. 5, 447-463.https://www.ncbi.nlm.nih.gov/pubmed/18018666

[6] D. Silove, Z. Steel, A. Bauman, T. Chey, A. McFarlane. Trauma, PTSD and the longer-term mental health burden amongst Vietnamese refugees. Social Psychiatry and Psychiatric Epidemiology, Vol. 42, No. 6, 467-476. https://www.ncbi.nlm.nih.gov/pubmed/18018666

[7] M. D. Morris, S. T. Popper, T. C. Rodwell, S. K. Brodine, K. C. Brouwer. Healthcare barriers of refugees post-resettlement. Journal of Community Health, Vol. 34, No. 6, 529-538 https://link.springer.com/article/10.1007/s10900-009-9175 $-3$ 
[8] P. Shannon, H. Im, E. Becher, J. Simmelink, E. Wieling, A, O'Fallon. Screening for war trauma, torture, and mental health symptoms among newly arrived refugees: A national survey of US refugee health coordinators. Journal of Immigrant \& Refugee Studies, Vol. 10, No. 4, 380-394. https://www.tandfonline.com/doi/abs/10.1080/15562948.2 012.674324

[9] M. S. Thomson, F. Chaze, U. George, S. Guruge. Improving immigrant populations' access to mental health services in Canada: A review of barriers and recommendations. Journal of Immigrant and Minority Health, Vol. 17, No. 6, 895-1905. https://link.springer.com/article/10.1007/s10903-015-0175 $-3$

[10] H. G. Rohlof, J. W. Knipscheer, R. J. Kleber. Somatization in refugees: A review. Social Psychiatry and Psychiatric Epidemiology, Vol. 49, No. 11, 1793-1804.https://link.springer.com/article/10.1007/s0012 7-014-0877-1

[11] C. Henning-Smith, T. P. Shippee, D. McAlpine, R. Hardeman, F. Farah. Stigma, discrimination, or symptomatology differences in self-reported mental health between US-born and Somalia-born Black Americans. American Journal of Public Health, Vol. 103, No. 5, 861-867.https://www.ncbi.nlm.nih.gov/pmc/articles/PMC3 $698822 /$

[12] S. Johnsdotter, K. Ingvarsdotter, M. Östman, A. Carlbom. Koran reading and negotiation with jinn: Strategies to deal with mental ill health among Swedish Somalis. Mental Health,Religion \& Culture, Vol. 14, No. 8, 741-755.https://www.tandfonline.com/doi/abs/10.1080/13 674676.2010 .521144

[13] J. E. Bettmann, D. Penney, P. Clarkson Freeman, N. Lecy. Somali refugees' perceptions of mental illness. Social Work in Health Care. Vol. 54, No. 8, 738-57.https://www.tandfonline.com/doi/full/10.1080/009 81389.2015.1046578

[14] S. May, R. M. Rapee, M. Coello, S. Momartin, J. Aroche. Mental health literacy among refugee communities: Differences between the Australian lay public and the Iraqi and Sudanese refugee communities. Social Psychiatry and Psychiatric Epidemiology, Vol. 49, No. 5, 757-769. https://www.ncbi.nlm.nih.gov/pubmed/24248469

[15] N. Almoshmosh. Highlighting the mental health needs of Syrian refugees. Intervention, Vol. 13, No. 2, 178-181. https://www.interventionjournal.com/sites/default/files/Hig hlighting_the_mental_health_needs_of_Syrian.8.pdf

[16] H. Y. Lee, K. Lytle, P. N. Yang, T. Lum. Mental health literacy in Hmong and Cambodian elderly refugees: A barrier to understanding, recognizing, and responding to depression. The International Journal of Aging and Human Development, Vol. 71, No. 4, 323-344.https://www.ncbi.nlm.nih.gov/pubmed/21261138

[17] H. De Anstiss, T. Ziaian. Mental health help-seeking and refugee adolescents: Qualitative findings from a mixed-methods investigation. Australian Psychologist, Vol. 45, No. $\quad$, 29-37.https://aps.onlinelibrary.wiley.com/doi/abs/10.1080/ 00050060903262387

[18] P. J. Shannon, E. Wieling, J. Simmelink-McCleary, E.
Becher. Beyond stigma: Barriers to discussing mental health in refugee populations. Journal of Loss and Trauma, Vol. 20, No. 281-296.https://psycnet.apa.org/record/2015-14708-008

[19] M. G. U. Guajardo, S. Slewa-Younan, Y. Santalucia, A. F. Jorm. Important considerations when providing mental health first aid to Iraqi refugees in Australia: A Delphi study. International Journal of Mental Health Systems, Vol. 10, No. $\quad 1, \quad 54-62$. https://www.ncbi.nlm.nih.gov/pubmed/27594898

[20] R. Asgary, N. Segar. Barriers to health care access among refugee asylum seekers. Journal of Health Care for the Poor and Underserved, Vol. 22, No. 2, 506-522.https://www.ncbi.nlm.nih.gov/pubmed/21551930

[21] N. V. Ismayilov, I. Fuad. Mental health of refugees: The case of Azerbaijan. World Psychiatry, Vol. 1, No. 2, 121-122.

https://www.ncbi.nlm.nih.gov/pmc/articles/PMC1489868/

[22] P. Subedi, C. Li, A. Gurung, D. Bizune, M. C. Dogbey, C. C. Johnson, K. Yun. Mental health first aid training for the Bhutanese refugee community in the United States. International Journal of Mental Health Systems, Vol. 9, No. 1 , 20-26.https://link.springer.com/article/10.1186/s13033-01 $5-0012-\mathrm{z}$

[23] A. F. Collier, M. Munger, Y. K. Moua, Y.K. Hmong mental health needs assessment: A community-based partnership in a small mid-western community. American Journal of Community Psychology, Vol. 49, No. 1-2, 73-86. https://link.springer.com/article/10.1007/s10464-011-9436 $-\mathrm{Z}$

[24] T. T. Donnelly, J. J. Hwang, D. Este, C. Ewashen, C. Adair, M. Clinton. If I was going to kill myself, I wouldn't be calling you. I am asking for help: Challenges influencing immigrant and refugee women's mental health. Issues in Mental HealthNursing, Vol. 32, No. 5, 279-290.https://www.tandfonline.com/doi/full/10.3109/01 612840.2010 .550383

[25] C. L. Tobin, P. Di Napoli, C. T. Beck. Refugee and immigrant women's experience of postpartum depression: A meta-synthesis. Journal of Transcultural Nursing, Vol. 29 , No.1, 84-100.https://journals.sagepub.com/doi/full/10.1177/1043 659616686167

[26] B. H. Ellis, A. K. Lincoln, M. E. Charney, R. Ford-Paz, M. Benson, L. Strunin. Mental health service utilization of Somali adolescents: Religion, community, and school as gateways to healing. Transcultural Psychiatry, Vol. 47, No. 5,789-811.https://journals.sagepub.com/doi/abs/10.1177/13 63461510379933

[27] P. J. Shannon. Refugees' advice to physicians: How to ask about mental health. Family practice, Vol. 31, No. 4, 462-466.https://academic.oup.com/fampra/article/31/4/46 2/710377

[28] M. Thikeo, P. Florin, C. Ng, C. Help seeking attitudes among Cambodian and Laotian refugees: Implications for public mental health approaches. Journal of immigrant and minority health, Vol. 17, No. 6, 1679-1686.https://link.springer.com/article/10.1007/s1090 3-015-0171-7 
[29] S. M. Weine. Developing preventive mental health interventions for refugee families in resettlement. Family Process, Vol. 50, No. 3, 410-430.https://onlinelibrary.wiley.com/doi/abs/10.1111/j. 1545-5300.2011.01366.x

[30] K. Polcher, S. Calloway. Addressing the need for mental health screening of newly resettled refugees: A pilot project. Journal of Primary Care \& Community Health, Vol. 7 , No. 3,199-203.https://journals.sagepub.com/doi/full/10.1177/21 50131916636630

[31] B. A. Kohrt, S. M. Maharjan, D. Timsina, J. L. Griffith. Applying Nepali ethnopsychology to psychotherapy for the treatment of mental illness and prevention of suicide among Bhutanese refugees. Annals of Anthropological Practice, Vol.36, No. 1, 88-112.https://anthrosource.onlinelibrary.wiley.com/doi/fu 11/10.1111/j.2153-9588.2012.01094.x

[32] S. J. Gold. Cross-cultural medicine, a decade later: Mental health and illness in Vietnamese refugees. Western Journal of Medicine, Vol. 157, No. 3, 290-294.https://repository.library.georgetown.edu/handle/ $10822 / 857978$

[33] P. J. Shannon, G. A.Vinson, T. L. Cook, E. Lennon. Characteristics of successful and unsuccessful mental health referrals of refugees. Administration and Policy in Mental Health and Mental Health Services Research, Vol. 43 , 4,555-568.https://link.springer.com/article/10.1007/s10488 $-015-0639-8$

[34] J. M. O’Mahony, T. T. Donnelly, S. R. Bouchal, D. Este. Cultural background and socioeconomic influence of immigrant and refugee women coping with postpartum depression. Journal of Immigrant and Minority Health, Vol. 15 , No.

2 , 300-314.https://link.springer.com/article/10.1007/s10903012-9663-x

[35] D. Palmer, K. Ward. 'Lost': listening to the voices and mental health needs of forced migrants in London. Medicine, Conflict and Survival, Vol. 23, No. 3, 198-212.https://www.tandfonline.com/doi/full/10.1080/13 623690701417345

[36] T. Ao, E. Taylor, E. Lankau, T. I. Sivilli, C. Blanton, S. Shetty, B. Lopes-Cardozo. An investigation into suicides among Bhutanese refugees in the us 2009-2012 stakeholders report. Center for Disease Control and Prevention.http://refugeehealthta.org/wp-content/uploads/2 012/10/Bhutanese-Suicide-Stakeholder Report_October 2 2_2012_Cleared_-For_Dissemination2-1.pdf

[37] B. H. Ellis, A. B. Miller, H. Baldwin, S. Abdi. New directions in refugee youth mental health services: Overcoming barriers to engagement. Journal of Child \& Adolescent Trauma, Vol. 4, No. 1, 69-85.https://www.tandfonline.com/doi/full/10.1080/1936 1521.2011 .545047

[38] M. Fazel, J. Garcia, A. Stein. The right location? Experiences of refugee adolescents seen by school-based mental health services. Clinical child psychology and psychiatry, Vol. 21, No. 3, 368-380.https://journals.sagepub.com/doi/full/10.1177/135 9104516631606
[39] M. Fazel, H. Doll, A. Stein. A school-based mental health intervention for refugee children: An exploratory study. Clinical Child Psychology and Psychiatry, Vol. 14, No. 2, 297-309.https://journals.sagepub.com/doi/abs/10.1177/13 59104508100128

[40] K. E. Miller. Rethinking a familiar model: Psychotherapy and the mental health of refugees. Journal of Contemporary Psychotherapy, Vol. 29, No. 4, 283-306.https://link.springer.com/article/10.1023/A:10229 26721458

[41] E. Gong-Guy, R. B. Cravens, T. E. Patterson. Clinical issues in mental health service delivery to refugees. American Psychologist, Vol. 46, No. 6, 642-648.https://psycnet.apa.org/record/1991-28488-001

[42] H. Jefee-Bahloul, M. Bajbouj, J. Alabdullah, G. Hassan, A. Barkil-Oteo. Mental health in Europe's Syrian refugee crisis. The Lancet Psychiatry, Vol. 3, No. 4, 315-317.https://www.thelancet.com/journals/lanpsy/article /PIIS2215-0366(16)00014-6/fulltext

[43] A. Ahmed, A. Bowen, C. X. Feng. Maternal depression in Syrian refugee women recently moved to Canada: A preliminary study. BMC Pregnancy and Childbirth, Vol. 17, No. 1 , 240 - 250 .https://bmcpregnancychildbirth.biomedcentral. com/articles/10.1186/s12884-017-1433-2

[44] C. E. Johnson-Agbakwu, J. Allen, J. F. Nizigiyimana, G. Ramirez, M. Hollifield. Mental health screening among newly arrived refugees seeking routine obstetric and gynecologic care. Psychological Services, Vol. 11, No. 4, 470-476. https://psycnet.apa.org/record/2014-45739-009

[45] B. D. M. Schuchman, C. McDonald. Somali mental health. American Journal of Public Health, Vol. 94, 591-598. https://ethnomed.org/clinical/mental-health/Somali-mental -health.pdf

[46] K. H. Nazzal, M. Forghany, M. C. Geevarughese, V. Mahmoodi, J. Wong. An innovative community-oriented approach to prevention and early intervention with refugees in the United States. Psychological Services, Vol. 11, No. 4, 477-485. https://psycnet.apa.org/record/2014-45739-010

[47] S. Guruge, A. Z. Y. Wang, V. Jayasuriya-Illesinghe, S Sidani, S. Knowing so much, yet knowing so little: A scoping review of interventions that address the stigma of mental illness in the Canadian context. Psychology, Health \& Medicine, Vol. 22, No. 5, 507-523.https://www.tandfonline.com/doi/abs/10.1080/13 548506.2016.1191655

[48] H. Stuart. Reducing the stigma of mental illness. Global Mental Health, Vol. 3, No. e17, 1-14. https://www.cambridge.org/core/journals/global-mental-he alth/article/reducing-the-stigma-of-mental-illness/261AB4 B0FCF056F137883350AF1133BF

[49] G. Thornicroft, N. Mehta, S. Clement, S. Evans-Lacko, M. Doherty, D. Rose, M. Koschorke, R. Shidhaye, C. O'Reilly, C. Henderson. Evidence for effective interventions to reduce mental-health-related stigma and discrimination. The Lancet, Vol. 387, No. 10023, 1123-1132.https://www.sciencedirect.com/science/article/p ii/S0140673615002986 
[50] L. Knifton, M. Gervais, K. Newbigging, N. Mirza, N. Quinn, N. Wilson, E. Hunkins-Hutchison. Community conversation: Addressing mental health stigma with ethnic minority communities. Social Psychiatry and Psychiatric Epidemiology, Vol. 45, No. 4, 497-504.https://link.springer.com/article/10.1007/s00127009-0095-4

[51] P. C. Gronholm, C. Henderson, T. Deb, G. Thornicroft. Interventions to reduce discrimination and stigma: The state of the art. Social Psychiatry and Psychiatric Epidemiology, Vol. 52, No. 3, 249-258.https://link.springer.com/article/10.1007/s00127$017-1341-9$

[52] G. Sampogna, I. Bakolis, S. Evans-Lacko, E. Robinson, G. Thornicroft, C. Henderson, C. The impact of social marketing campaigns on reducing mental health stigma: Results from the 2009-2014 time to change programme. European Psychiatry, Vol. 40 , 116-122.https://www.sciencedirect.com/science/article/pii/ S0924933816301109

[53] H. Bingham, A. J. O'Brien. Educational intervention to decrease stigmatizing attitudes of undergraduate nurses towards people with mental illness. International Journal of Mental Health Nursing, Vol. 27, No. 1, 311-319. https://onlinelibrary.wiley.com/doi/abs/10.1111/inm.12322

[54] M. Rubio-Valera, I. Aznar-Lou, M. Vives-Collet, A. Fernández, M. Gil-Girbau, A. Serrano-Blanco. Reducing the mental health-related stigma of social work students: A cluster RCT. Research on Social Work Practice, Vol. 28, No.

164-172.https://journals.sagepub.com/doi/full/10.1177/10 49731516641492

[55] S. P. Chen, M. Koller, T. Krupa, H. Stuart. Contact in the classroom: Developing a program model for youth mental health contact-based anti-stigma education. Community Mental Health Journal, Vol. 52, No. 3, 281-293. https://link.springer.com/article/10.1007/s10597015-9944-7

[56] J. K. Fokuo, V. Goldrick, J. Rossetti, C. Wahlstrom, C. Kocurek, J. Larson, P. Corrigan, P. Decreasing the stigma of mental illness through a student-nurse mentoring program: A qualitative study. Community Mental Health Journal, Vol. 53, No. 3, 257-265.https://link.springer.com/article/10.1007/s10597016-0016-4

[57] M. D. Pinto-Foltz, M. C. Logsdon, J. A. Myers. Feasibility, acceptability, and initial efficacy of a knowledge-contact program to reduce mental illness stigma and improve mental health literacy in adolescents. Social Science \& Medicine, Vol. 72, No. 12, 2011-2019.https://www.sciencedirect.com/science/article/ pii/S0277953611002425

[58] S. Evans-Lacko, J. London, S. Japhet, N. Rüsch, C. Flach, E. Corker, C. Henderson, G. Thornicroft. Mass social contact interventions and their effect on mental health related stigma and intended discrimination. BMC Public Health, Vol. 12, No.1, 489-496.https://bmcpublichealth.biomedcentral.com/articl es/10.1186/1471-2458-12-489

[59] A. H. Hackler, M. A. Cornish, D. L. Vogel. Reducing mental illness stigma: Effectiveness of hearing about the normative experiences of others. Stigma and Health, Vol. 1, No. 201-205. https://psycnet.apa.org/record/2016-07226-001

[60] M. Koller, H. Stuart. Reducing stigma in high school youth. Acta Psychiatrica Scandinavica, Vol. 134, 63-70. https://onlinelibrary.wiley.com/doi/full/10.1111/acp s. 12613

[61] S. E. Hanisch, C. D. Twomey, A. C. Szeto, U. W. Birner, D. Nowak, C. Sabariego. The effectiveness of interventions targeting the stigma of mental illness at the workplace: A systematic review. BMC Psychiatry, Vol.16, No. 1, 1-11. https://bmcpsychiatry.biomedcentral.com/articles/10.1186/ s12888-015-0706-4

[62] G. Schomerus, M. C. Angermeyer, S. E. Baumeister, S. Stolzenburg, B. G. Link, J. C. Phelan. An online intervention using information on the mental health-mental illness continuum to reduce stigma. European Psychiatry, Vol.

32,21-27.https://www.sciencedirect.com/science/article/pii /S0924933815006835

[63] A. Lucksted, A. Drapalski, C. Calmes, C. Forbes, B. DeForge, J. Boyd. Ending self-stigma: Pilot evaluation of a new intervention to reduce internalized stigma among people with mental illnesses. Psychiatric Rehabilitation Journal, Vol. $\quad 35, \quad$ No. 1 , 51-54.https://psycnet.apa.org/record/2011-15918-008

[64] P. T. Yanos, A. Lucksted, A. L. Drapalski, D. Roe, P. Lysaker. Interventions targeting mental health self-stigma: A review and comparison. Psychiatric Rehabilitation Journal, Vol. $38, \quad$ No. 171-178.https://psycnet.apa.org/record/2014-42856-001

[65] L. Wood, R. Byrne, F. Varese, A. P. Morrison. Psychosocial interventions for internalised stigma in people with a schizophrenia-spectrum diagnosis: A systematic narrative synthesis and meta-analysis. Schizophrenia Research, Vol. 176, No. 2-3, 291-303.https://www.sciencedirect.com/science/article/pii/ S0920996416302195

[66] B. D. Dickstein, D. S. Vogt, S. Handa, B. T. Litz. Targeting self-stigma in returning military personnel and veterans: A review of intervention strategies. Military Psychology, Vol. 22, No. 2, 224-236.https://www.tandfonline.com/doi/full/10.1080/08 995600903417399

[67] S. Yamaguchi, S. I. Wu, M. Biswas, M. Yate, Y. Aoki, E. A. Barley, G. Thornicroft. Effects of short-term interventions to reduce mental health-related stigma in university or college students: A systematic review. The Journal of Nervous and Mental Disease, Vol. 201, No. 6, 490-503.https://journals.lww.com/jonmd/FullText/2013/06 000/Effects_of_Short_Term_Interventions_to_Reduce.8.as $\mathrm{px}$

[68] N. Mehta, S. Clement, E. Marcus, A. C. Stona, N. Bezborodovs, S. Evans-Lacko, J. Palacios, M. Docherty, E. Barley, D. Rose, M. Koschorke. Evidence for effective interventions to reduce mental health-related stigma and discrimination in the medium and long term: Systematic review. The British Journal of Psychiatry, Vol. 207, No. 5, 377-384.

https://www.cambridge.org/core/journals/the-british-journa l-of-psychiatry/article/evidence-for-effective-interventions- 
to-reduce-mental-healthrelated-stigma-and-discriminationin-the-medium-and-long-term-systematic-review/2C54EC CA361ECF5916D96D7EA133A165

[69] A. J. Morgan, N. J. Reavley, A. Ross, L. San Too, A. F. Jorm. Interventions to reduce stigma towards people with severe mental illness: Systematic review and meta-analysis. Journal of Psychiatric Research, Vol. 103, 120-133. https://www.sciencedirect.com/science/article/pii/S002239 5618303558

[70] P. W. Corrigan, D. Rao, D. On the self-stigma of mental illness: Stages, disclosure, and strategies for change. The Canadian Journal of Psychiatry, Vol. 57, No. 8, 464-469. https://journals.sagepub.com/doi/abs/10.1177/0706743712 05700804

[71] K. M. Griffiths, B. Carron - Arthur, A. Parsons, R. Reid. Effectiveness of programs for reducing the stigma associated with mental disorders. A meta-analysis of randomized controlled trials. World Psychiatry, Vol. 13, No.

161-175.https://onlinelibrary.wiley.com/doi/full/10.1002/ wps.20129

[72] A. Milner, P. C. F. Law, C. Mann, T. Cooper, K. Witt, A. D. LaMontagne. A smart-phone intervention to address mental health stigma in the construction industry: A two-arm randomised controlled trial. SSM-Population Health, Vol. 4 164-168.https://www.sciencedirect.com/science/article/pii /S235282731730215X

[73] L. Hansson, A. Lexén, J. Holmén. The effectiveness of narrative enhancement and cognitive therapy: A randomized controlled study of a self-stigma intervention. Social Psychiatry and Psychiatric Epidemiology, Vol. 52, No.

11,1415-1423.https://link.springer.com/article/10.1007/s00 127-017-1385-x

[74] R. B. Büchter, M. Messer. Interventions for reducing self-stigma in people with mental illnesses: A systematic review of randomized controlled trials. GMS German Medical Science, $\quad$ Vol. 15 , 1-12.https://www.ncbi.nlm.nih.gov/pmc/articles/PMC5404 $117 /$ 